\title{
Can silver nanoparticles affect the mineral content, structure and mechanical properties of chicken embryo bones?
}

\author{
J. Sikorska ${ }^{1,4}$, M. Szmidt ${ }^{2}$, E. Sawosz ${ }^{1}$, T. Niemiec ${ }^{1}$, M. Grodzik ${ }^{1}$ \\ and A. Chwalibog ${ }^{3}$ \\ Warsaw University of Life Sciences, \\ ${ }^{1}$ Department of Animal Nutrition and Feed Science \\ ${ }^{2}$ Faculty of Veterinary Medicine, Department of Morphologic Sciences \\ Ciszewskiego 8, 02-786 Warsaw, Poland \\ ${ }^{3}$ University of Copenhagen, Department of Basic Animal and Veterinary Sciences \\ Groennegaardsvej 3, 1870 Frederiksberg C, Denmark
}

(Received 12 January 2010; revised version 26 March 2010; accepted 9 May 2010)

\begin{abstract}
Biomedical application of silver nanoparticles has recently gained much attention. In this study, we investigated whether hydrocolloids of silver nanoparticles (nano-Ag) are deposited in chicken bones during embryogenesis and to what extent they can affect biochemical characteristics, mineral content, structure, and mechanical properties of bones. Hydrocolloids $(0.3 \mathrm{ml})$, containing $50 \mathrm{ppm}$ of nano-Ag, were injected in ovo prior to incubation of eggs. After 20 days of incubation, blood and thigh bones were isolated and analysed. Nano-Ag was deposited in embryo thigh bones, but did not affect the structure or mechanical properties of the bone. There was no effect of nano-Ag on the selected biochemical indices, but there was a tendency towards increasing mineral content, indicating that nanoparticles may influence bone mineralization. Considering that nano-Ag is absorbed by the embryo skeleton without affecting bone properties, these particles might be a good candidate for carriers of micronutrients or drugs into bones.
\end{abstract}

KEY WORDS: nanoparticles, silver, bones, mineral content, mechanical properties, chicken embryo

\section{INTRODUCTION}

In recent years silver nanoparticles have emerged in a variety of biomedical applications (Salata, 2004; Bhattacharya and Mukherjee, 2008; Rai et al., 2009).

\footnotetext{
* Supported by Danish Agency for Science, Technology and Innovation, Grant 2106-08-0025

These results are part of the Thesis of Justyna Sikorska

${ }^{4}$ Corresponding author: e-mail: justyna_sikorska@sggw.pl
} 
Ag nanoparticles (nano-Ag) possess both characteristics of the chemically neutral metal and a substance with a high level of nanoscale fragmentation. Nanoscale together with an enormous surface, lead to the biological activity of nanoparticles even in very low concentrations (Williams, 2002). The mechanism of this bioactivity is not associated with chemical reactions but with the ability to carry, store and interact bio-electrically (Nagy et al., 1999).

Recently, it has been demonstrated that nano-Ag does not affect growth, development, or DNA oxidative damage in chicken embryos (Sawosz et al., 2009). In rat studies, animals exposed to different doses of nano-Ag did not alter body and organ weights, blood biochemistry, haematological characteristics and did not cause bone marrow cytotoxicity. Elevated levels of alkaline phosphatase were observed, however (Kim et al., 2008). The enzyme is associated with bone formation and it is present in the bone ossification centre before hypertrophic chondrocytes become present. Furthermore, the content of silver in testes, kidneys, liver, brain, lungs and stomach increased with increasing levels of nano-Ag (Kim et al., 2008), suggesting that this could also be the case for bones. Nevertheless, no data is available regarding the nano-Ag content in bones, either during prenatal or postnatal development. The key factor in chicken development is proper nutrition during embryogenesis (Foye et al., 2006). The main source of calcium for the growing embryo is its eggshell (Johnston and Comar, 1955). Thanks to the chorioallantoic membrane releasing acids, which dissolve the eggshell, the availability of the elements is markedly elevated (Leeson and Leeson, 1963). We hypothesized that nano-Ag can be retained in bones as in other tissues and may have an effect on bone mineralization and development during embryogenesis. This novel approach has not been presented to date. Thus, the objective of the present study was to determine nano-Ag deposition in chicken embryo bones and its potential effect on the mineral content, structure and mechanical properties of bones, and on selected biochemical characteristics.

\section{MATERIAL AND METHODS}

The experimental factor used in our analysis was a hydrocolloid of silver nanoparticles prepared with deionized water (Nano-Tech, Polska). The Ag nanoparticles were produced with non-combustible metal of the highest purity. Inseminated eggs of the Ross 308 meat chicken $(n=120)$ were obtained from the Institute of Poultry Farming, Dębówka (Poland) and stored for 4 days at $12^{\circ} \mathrm{C}$. The eggs were weighed and randomly divided into three groups with 40 eggs of equal weight $(60.4 \pm 2.8 \mathrm{~g})$ per group. The eggshell was washed with $0.05 \%$ potassium permanganate $\left(\mathrm{KMnO}_{4}\right)$ at the point of injection $(2-3 \mathrm{~cm}$ from the sharp end of the egg). The incision in the shell was performed with a sterile scalpel and $300 \mu \mathrm{l}$ of the experimental solution were injected with a disposable needle. In the placebo group, $0.9 \% \mathrm{NaCl}$ was injected into the egg. The eggs were incubated to 
the $20^{\text {th }}$ day of embryonic development under standard conditions with constant temperature, humidity, and egg rotation (ALMD1 N5 FUH Walenski). Normal conditions $\left(37.7{ }^{\circ} \mathrm{C}, 60 \%\right.$ humidity and rotation every $\left.60 \mathrm{~min}\right)$ were applied until the $18^{\text {th }}$ day of incubation. During the last two days of incubation, the eggs were kept at $37^{\circ} \mathrm{C}$ and $70 \%$ humidity with no rotation. Embryos 20 days of age were sacrificed by decapitation. Blood and thigh bone were isolated from each animal and stored at $-20^{\circ} \mathrm{C}$. Each thigh bone was analysed for its calcium, magnesium, iron, copper, zinc and silver contents. The procedure was performed using a laboratory Ethos $\mathrm{D}$ microwave system (Milestone). Specimens were placed in high-pressure Teflon dishes and burned with the addition of $5 \mathrm{ml} \mathrm{HNO}_{3}$ (Merck) and $1 \mathrm{ml} \mathrm{H}_{2} \mathrm{O}_{2}$ (Merck). The concentration of the elements was analysed with an ICP-MS spectrophotometer (ELAN DRC II, Perkin Elmer). The results for calcium and magnesium are presented as $\mathrm{mg} / 100 \mathrm{~g}$ of tissue, those for the other elements, as $\mathrm{mg} / \mathrm{kg}$.

The concentrations of blood serum biochemical characteristics like alkaline phosphatase, $\mathrm{Ca}, \mathrm{P}, \mathrm{Mg}$ were measured by the biochemical analyzer Vitros DT 60 II (modules DT, DTE, DTSC) and ready-to-use slide kits (Johnson and Johnson Ortho-Clinical Diagnostics). The enzyme results are presented in U/l and mineral contents are shown in $\mathrm{mmol} / \mathrm{l}$.

Mechanical parameters of the bone were analysed using an INSTRON 4301 apparatus. Tensile strength was measured by applying pressure to the bone's long axis with at a speed of $13 \mathrm{~mm} / \mathrm{min}$ and force of $10 \mathrm{~N}$. Maximal pressure before breaking and breaking tension $(\mathrm{Pa})$ were recorded. Estimation of the spongy bone trabecular structure was performed in the bone cross section with the use of an SEM Jeol JSM-35 (XT Microscope Control Quanta ver. 2.3) scanning electron microscope at a magnification of 50 and 1500 times.

The data were analysed using mono-factorial analysis of variance ANOVA and the differences between groups were tested by the multiple range Duncan test, using Statgraphics Plus 4.1. Differences with $\mathrm{P}<0.05$ were considered significant.

\section{RESULTS AND DISCUSSION}

In the present experiment the content of nano- $\mathrm{Ag}$ was $0.25 \mu \mathrm{g} \mathrm{Ag} / \mathrm{g}$ egg. Considering that the average weight of bones in the nano-Ag group was $0.22 \mathrm{~g}$, about $8 \%$ of the molecular silver administered to the eggs was retained in the thigh bone, which clearly indicates deposition of nano-Ag in the embryo's skeleton.

Nano-Ag did not significantly affect the thigh bone mineral content of the examined chicken embryos (Table 1), although compared with the control group, the embryos treated with $\mathrm{Ag}$ tended to have a higher Ca content $(3 \%)$ and increased contents of iron (12\%) and copper (9\%). Copper is a necessary element for the synthesis of bone tissue collagen network, acting together with vitamin $\mathrm{B}_{6}$ (pyridoxine) as a cofactor of inter- and intra-molecular covalent bond formation (Libby and Aikawa, 2002). The increased content of $\mathrm{Cu}$ improves bone elastic 
Table 1. Mineral content of chicken embryo's thigh bone

\begin{tabular}{cccccc}
\hline Parameters & Control & Placebo & Nano-Ag & SE pooled & P-value \\
\hline $\mathrm{mg} / 100 \mathrm{~g}$ & & & & \\
$\mathrm{Ca}$ & 8.48 & 8.91 & 8.76 & 0.754 & 0.64 \\
$\mathrm{Mg}$ & 0.21 & 0.21 & 0.22 & 0.021 & 0.96 \\
$\mathrm{mg} / \mathrm{kg}$ & \multicolumn{5}{c}{} \\
$\mathrm{Fe}$ & 200.0 & 209.2 & 223.8 & 21.63 & 0.44 \\
$\mathrm{Cu}$ & 3.39 & 3.27 & 3.69 & 0.550 & 0.45 \\
$\mathrm{Zn}$ & 98.7 & 91.7 & 93.4 & 13.971 & 0.75 \\
$\mathrm{Ag}$ & $0.00^{\mathrm{a}}$ & $0.00^{\mathrm{a}}$ & $0.09^{\mathrm{b}}$ & 0.040 & 0.001 \\
\hline $\mathrm{a}, \mathrm{b}$ & values within rows with different superscripts are significantly different, $\mathrm{P}<0.05$
\end{tabular}

characteristics (Gralak et al., 2004), while its deficiency leads to rickets, deformation, or excessive brittleness (Puls, 1995).

Nano-Ag did not affect the selected blood biochemical parameters (Table 2), indicating that the treatment did not influence related metabolic pathways. In the nano$\mathrm{Ag}$ treated group, we observed a tendency towards an increasing concentration of $\mathrm{Ca}$

Table 2. Biochemical parameters of chicken embryo's blood serum

\begin{tabular}{lccccc}
\hline Parameters & Control & Placebo & Nano-Ag & SE pooled & P-value \\
\hline Alkaline phosphatase, U/1 & 781.7 & 672.7 & 704.4 & 205.020 & 0.34 \\
Minerals, mmol/1 & & & & & \\
$\quad \mathrm{Ca}$ & 2.17 & 2.34 & 2.55 & 0.521 & 0.08 \\
$\mathrm{P}$ & 1.99 & 1.69 & 1.73 & 0.453 & 0.30 \\
$\mathrm{Mg}$ & 0.72 & 0.76 & 0.75 & 0.165 & 0.32 \\
\hline
\end{tabular}

significant when $\mathrm{P}<0.05$

ions in blood serum. Bone mineralization is a complex process requiring a specific concentration of Ca ions in the body fluids. Under physiological conditions, however, the level of ion activation is below the value causing spontaneous precipitation of calcium phosphates. Consequently, initiation of bone mineralization requires energy and other factors necessary for formation of the crystal primary nucleus. First, the primary nucleus ions can accrete into bigger crystals. Such an increase of crystals can be a source of nucleus mineralization (Dziedzic-Gocławska, 1995). It can be speculated that nano-Ag has the ability to stimulate the hydroxylapatite formation.

The mean maximum value of pressures before breaking and the breaking tension were similar in all groups (Table 3), indicating no negative effects of nanoAg on these mechanical characteristics. Physically, bone structure is reinforced by the network of spongy bone trabeculae, which allows maximal efficiency during load carrying (Currey, 2004a). Bone tissue is built from two main phases: organic-like collagen fibres and mineral-like hydroxylapatite crystals. The mineral phase affects, first of all, elasticity while the fibres affect plasticity. Thus, bone tissue breaking strength depends on the quality and proportion between 
Table 3. Mechanical properties of chicken embryo's thigh bone

\begin{tabular}{lccccc}
\hline Parameters & Control & Placebo & Nano-Ag & SE pooled & P-value \\
\hline F max, N & 5.28 & 5.06 & 5.22 & 0.310 & 0.88 \\
Tensile strength, $\mathrm{Pa}$ & 0.62 & 0.59 & 0.63 & 0.044 & 0.86 \\
\hline
\end{tabular}

significant when $\mathrm{P}<0.05$

the phases (Szulc et al., 2005). The present values of mechanical characteristics demonstrated that nano-Ag had no effect on bone elasticity or plasticity. When discussing bone breaking strength the important aspect is the concept of point of inertia, being dependent on the shape of the section related to the control axis of force interaction. With age, diaphysis long bone diameters increase, causing an elevation of bone point of inertia (Currey, 2004 b). The structure and size of the spongy bone were evaluated by a scanning electron microscope (SEM). Inspection of thigh bone cross sections (Picture 1) led to the conclusion that the bone structure was not damaged or weakened by nano-Ag.

Increased magnification of SEM images showed that the amount of bone plaques and trabeculae was significantly higher than in bones in the control group. Specific mechanical functions also depend on the direction of bone trabeculae, which are usually related to the lines of pressure forces. The images presented, in all groups, bone diaphysis as hollow cylinders with similar diameters. The space in between the spongy bone trabeculae was filled by the bone marrow.

It can be concluded that nanoparticles of silver were deposited in embryo thigh bones but did not affect bone structure or mechanical properties. There was no effect.

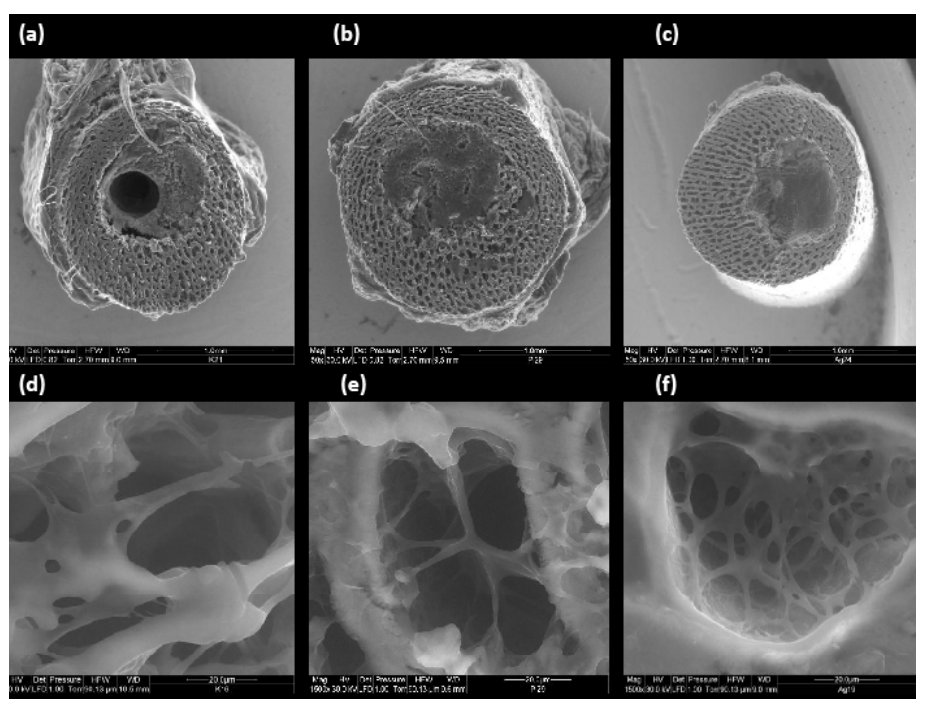

Figure 1. SEM micrograph of femur from chicken embryos; (a), (b), (c) - magnification 50; (d), (e), (f) - magnification 1500, (a), (d) - Control; (b), (e) - Placebo; (c), (f) - Nano-Ag 
of nano-Ag on the chosen biochemical indices, but, there was a tendency towards an increasing content of calcium, iron and copper, indicating that nano-Ag may influence bone mineralization. Considering that there is no available literature dealing with nano-Ag and bone characteristics during embryogenesis, possible effects on bone mineralization require further research. Furthermore, considering that nano-Ag is absorbed by the chicken skeleton without affecting structure, mechanical properties, or biochemical characteristics, it might be a potential carrier of micronutrients or drugs into bones.

\section{REFERENCES}

Bhattacharya R., Mukherjee P., 2008. Biological properties of „naked” metal nanoparticles. Advan. Drug Delivery Rev. 60, 1289-1306

Currey D.J., 2004a. Tensile yield in compact bone is determined by strain, post-yield behaviour by mineral content. J. Biomech. 37, 549-556

Currey D.J., 2004b. Incompatible mechanical properties in compact bone. Theor. Biol. 231, 569-580

Dziedzic-Gocławska A., 1995. Bone tissue (in Polish). In: Histology. PZWL, pp. 244-305

Foye O.T., Uni Z., Ferket P.R., 2006. Effect of in ovo feeding egg white protein, beta-hydroxy-betamethylbutyrate, and carbohydrates on glycogen status and neonatal growth of turkeys. Poultry Sci. 85, 1185-1192

Gralak M.A., Piastowska A.W., Leontowicz H., Leontowicz M., Antczak A., Kulasek G., Szara T., Narojek T., 2004. Effect of dietary protein level and sources on bone mineralization and structure in rats. Biofactors 22, 25-28

Johnston P., Comar C., 1955. Distribution of calcium from the albumen, yolk and shell to the developing chick embryo. Amer. J. Physiol. 183, 365-370

Kim Y.S., Kim J.S., Cho H.S., Rha D.S., Kim J.M., Park J.D., Choi B.S., Lim R., Chang H.K., Chung Y.H., Kwon I.H., Jeong J., Han B.S., Yu I.J., 2008. Twenty-eight-day oral toxicity, genotoxicity, and gender-related tissue distribution of silver nanoparticles in Sprague-Dawley rats. Inhal. Toxicol. 20, 575-583

Leeson T., Leeson C., 1963. The chorioallantoic of the chick. Light and electron microscopic observations at various times of incubation. J. Anat. 97, 585-559

Libby P., Aikawa M., 2002. Vitamin C, collagen, and cracks in the plaque. Circulation 105, 1396-1398

Nagy A.J., Mestl G., Herein D., Weinberg G., Kitzelmann E., Schlögl R., 1999. The correlation of subsurface oxygen diffusion with variations of silver morphology in the silver-oxygen system. J. Catal. 182, 417-429

Puls R., 1995. Mineral Levels in Animal Health. Diagnostic Data. Serpa International, Clearbrook

Rai M., Yadav A., Gade A., 2009. Silver nanoparticles as a new generation of antimicrobials. Biotech. Adv. 27, 76-83

Salata O.V., 2004. Application of nanoparticles in bio0logy and medicine. J. Nanobiotech. 2, 1-6

Sawosz E., Grodzik M., Zielińska M., Niemiec T., Olszańska B., Chwalibog A., 2009. Nanoparticles of silver do not affect growth, development and DNA oxidative damage in chicken embryos. Arch. Geflügelk. 73, 208-213

Szulc P., Beck T.J., Marchand F., Delmas P.D., 2005. Low skeletal muscle mass is associated with poor structural parameters of bone and impaired balance in elderly men - The MINOS study. J. Bone Miner. Res. 20, 721-729

Williams D., 2002. Medical technology: How small we can go? Med. Device Tech. 4, 7-9 